\title{
Monodromy Inflation in the Strong Coupling Regime of the Effective Field Theory
}

\author{
Guido D’Amico, ${ }^{1, *}$ Nemanja Kaloper, ${ }^{2, \dagger}$ and Albion Lawrence ${ }^{3, \$}$ \\ ${ }^{1}$ Theoretical Physics Department, CERN, Geneva, Switzerland \\ ${ }^{2}$ Department of Physics, University of California, Davis, California 95616, USA \\ ${ }^{3}$ Martin Fisher School of Physics, Brandeis University, Waltham, Massachusetts 02453, USA
}

(Received 28 November 2017; revised manuscript received 14 June 2018; published 30 August 2018)

\begin{abstract}
We present a simple effective field theory formulation of a general family of single-field flux monodromy models for which strong coupling effects at large field values can flatten the potential and activate higher-derivative operators. Both of these effects can suppress the tensor amplitude. These models are radiatively and nonperturbatively stable and can sustain $\gtrsim 60 e$ folds of inflation. The dynamics combines features of both large-field chaotic inflation and $k$ inflation. Reducing the tensor-scalar ratio below the observational bound $r \lesssim 0.1$ while keeping the scalar spectral index $n_{s}$ within experimental bounds either yields equilateral non-Gaussianity $f_{\mathrm{NL}}^{\mathrm{eq}} \simeq \mathcal{O}(1)$, close to the current observational bounds, or gives very small $r$.
\end{abstract}

DOI: 10.1103/PhysRevLett.121.091301

Models of inflation with super-Planckian inflaton ranges can be remarkably simple and predictive [1,2]. They give the largest possible primordial tensor fluctuations, potentially detectable by $\mathrm{CMB}$ polarization experiments. However, control over the relevant $\geq m_{\mathrm{Pl}}$ field range requires additional model-building input. A promising control mechanism is monodromy [3-6]. In recent work, two of us proposed that generic monodromy inflation models have a simple field theory formulation based on a massive 4-form gauge theory [7] (see also Refs. [8,9]). The gauge symmetries - a compact $U(1)$ symmetry for the 4-form and a discrete phase space gauge symmetry for the dual scalar-protect slow-roll inflation from both perturbative and nonperturbative corrections.

The gauge field mass $m$ is a dimensionful coupling which controls the theory below the cutoff $M$. It appears in the combination $m \phi$, with $\phi$ as the inflaton field, so we take $\left[(m \phi) / M^{2}\right]$ as a dimensionless coupling up to factors of 2 and $\pi$. A weakly coupled theory produces a canonical kinetic term and quadratic potential [5-7,10], predicting a tensor-to-scalar ratio $r \sim 0.16$, ruled out by the current upper bound $r \lesssim 0.1$. In this Letter we discuss the strong coupling regime of the EFT [7], using naive dimensional analysis (NDA) [11,12] to write down the most general gauge-invariant, ghost-free EFT for energies below the cutoff scale $M$. Factors of $4 \pi$ open up an interval of strong coupling below the cutoff $M$, with irrelevant operators

Published by the American Physical Society under the terms of the Creative Commons Attribution 4.0 International license. Further distribution of this work must maintain attribution to the author(s) and the published article's title, journal citation, and DOI. Funded by SCOAP. comparable to marginal and relevant couplings, while slow roll remains protected from UV physics.

Strong coupling provides at least two significant effects: nontrivial "flattened" potentials as seen in string theory constructions $[3,4,13,14]$, and higher-derivative terms such as those in $k$ inflation $[15,16]$. We identify two dynamically and observationally distinct slow-roll inflationary phases. In one phase, the higher-derivative terms are dynamically suppressed, and inflation is the standard potential-driven slow roll. Either flattening provides a reduction of tensor power while increasing the spectral index $n_{s}$, or the potential plateaus out $[17,18]$, in which case $n_{s}$ remains close to the observed value, but $r$ is at most at the edge of detectability and the field range is at most $\mathcal{O}\left(m_{\mathrm{Pl}}\right)$. In the other phase, the higher-derivative terms are important, suppress the tensor power, and enhance "equilateral" non-Gaussianity to $f_{\mathrm{NL}}^{\mathrm{eq}} \simeq \mathcal{O}(1)$, close to the current observational bounds. In this class, bounds on $f_{\mathrm{NL}}^{\mathrm{eq}}$ imply a lower limit on $r$.

Which phase dominates in a given model, and how a transition from one to another can occur, is a matter for future work. Here we will study the phases separately and explore the parameter ranges consistent with observations and naturalness.

The low-energy EFT of Ref. [7] is the most general massive Abelian 3-form gauge theory, including all the irrelevant operators allowed by gauge symmetry. The action is

$$
\begin{aligned}
\mathcal{L}^{(\mathrm{full})}= & -\frac{1}{48} F_{\mu \nu \lambda \sigma}^{2}-\frac{m^{2}}{12}\left(A_{\mu \nu \lambda}-h_{\mu \nu \lambda}\right)^{2} \\
& -\sum_{n>2} \frac{a_{n}^{\prime}}{M^{2 n-4}} \tilde{F}^{n}-\sum_{n>1} \frac{a_{n}^{\prime \prime}}{M^{4 n-4}} m^{2 n}\left(A_{\mu \nu \lambda}-h_{\mu \nu \lambda}\right)^{2 n} \\
& -\sum_{k \geq 1, l \geq 1} \frac{a_{k, l}^{\prime \prime \prime}}{M^{4 k+2 l-4}} m^{2 k}\left(A_{\mu \nu \lambda}-h_{\mu \nu \lambda}\right)^{2 k} \tilde{F}^{l}
\end{aligned}
$$


(using the equations of motion to eliminate terms with $\partial^{k} F$ factors), where $A$ is the gauge field 3-form, $F=d A, \tilde{F}={ }^{*} F$, $b$ is a 2-form Stuickelberg gauge field with field strength $h=d b$, and $m$ plays the role of both the gauge field mass and the Stückelberg mode coupling. By gauge symmetry and the Goldstone boson equivalence theorem (GBET), any power of $A$ not covered by a derivative must be multiplied by the same power of $m$ [7].

To best study inflationary dynamics, we will work in the dual frame in which the longitudinal mode of $F$ is replaced by a compact scalar: schematically, $F \sim \epsilon(m \phi+Q), m A \sim \epsilon \partial \phi$ [7]. Here $Q=N q$, where $q$ is the fundamental 4-form charge, $N \in \mathbb{Z}$, and $\phi \equiv \phi+f$, where $m f=q[6]$. We will use the NDA framework to provide a more refined description of the dimensionless coefficients in Eq. (1): there are crucial factors of $1 / 4 \pi$ ensuring that the EFT including loop corrections below the cutoff $M$ is natural $[11,12]$. Such factors will give us a substantial inflaton range at strong coupling. The rules are as follows: (1) Replace $\phi$ with the dimensionless quantity $[(4 \pi \phi) / M]$. (2) Normalize the Lagrangian by $\left[M^{4} /(4 \pi)^{2}\right]$. (3) Include factorials in the denominators of Eq. (1) to account for the symmetry factors in the physical $S$-matrix elements. The upshot is that the strong coupling scale is $M_{s}=M / \sqrt{4 \pi}$, above which irrelevant operators can be activated without also activating the UV degrees of freedom (d.o.f.). This factor-of- $\sqrt{4 \pi}$ difference between the strong coupling scale and the cutoff is extremely important, since it prolongs the validity of EFT after the onset of strong coupling. This is particularly vivid when one considers high-dimension operators whose normalizations involve large powers of $1 / \sqrt{4 \pi}$ which can be much smaller than unity. The resulting effective Lagrangian, valid to the scale $M$, is

$$
\begin{aligned}
\mathcal{L}= & -\frac{1}{2}\left(\partial_{\mu} \phi\right)^{2}-\frac{1}{2}(m \phi+Q)^{2} \\
& -\sum_{n>2} c_{n}^{\prime} \frac{(m \phi+Q)^{n}}{n !\left(\frac{M^{2}}{4 \pi}\right)^{n-2}}-\sum_{n>1} c_{n}^{\prime \prime} \frac{\left(\partial_{\mu} \phi\right)^{2 n}}{2^{n} n !\left(\frac{M^{2}}{4 \pi}\right)^{2 n-2}} \\
& -\sum_{k \geq 1, l \geq 1} c_{k, l}^{\prime \prime \prime} \frac{(m \phi+Q)^{l}}{2^{k} k ! l !\left(\frac{M^{2}}{4 \pi}\right)^{2 k+l-2}}\left(\partial_{\mu} \phi\right)^{2 k},
\end{aligned}
$$

where $c_{n} \sim \mathcal{O}(1)$ by naturalness of the strong coupling expansion, absent additional symmetries. [Again, we remove $\partial^{k} \phi$ terms via the equations of motion. The $c_{n}$ 's depend on $m \phi+Q$ through the RG logs. At large $n$, they may grow as $\sim n$ ! by proliferation of graphs with fixed external lines, such that Eq. (2) is not an analytic function for all values of $\phi, X$.] Next, we define the inflaton as $\varphi=\phi+Q / m$. Since $Q=$ $2 \pi N q$ with $N \in Z, \varphi$ can vary over a range $\gg m_{\mathrm{Pl}}$, while all fundamental parameters including $f$ are below $m_{\mathrm{Pl}}$. The action (2) can then be formally written as

$$
\begin{aligned}
\mathcal{L} & =K(\varphi, X)-V_{\text {eff }}(\varphi) \\
& =\frac{M^{4}}{16 \pi^{2}} \mathcal{K}\left(\frac{4 \pi m \varphi}{M^{2}}, \frac{16 \pi^{2} X}{M^{4}}\right)-\frac{M^{4}}{16 \pi^{2}} \mathcal{V}_{\text {eff }}\left(\frac{4 \pi m \varphi}{M^{2}}\right),
\end{aligned}
$$

where $X \equiv-\left(\partial_{\mu} \varphi\right)^{2}$, and $\mathcal{K}, \mathcal{V}_{\text {eff }}$ should be understood as asymptotic series, well approximated by finitely many terms. This is precisely the form of the action of the $k$-inflation model of Refs. [15,16], with $\varphi$ and $X$ normalized by $\left[(4 \pi m) / M^{2}\right]$ and $\left[\left(16 \pi^{2}\right) / M^{4}\right]$, respectively. It is the natural EFT for single-field monodromy inflation.

Equation (3) supports two distinct phases. In the first, the terms nonlinear in $X$ are dynamically suppressed, while the third term can sum to a flattened potential supporting standard potential-driven slow roll. The effective action for $\varphi$ is

$\mathcal{L}=-\frac{1}{2} \mathcal{Z}_{\text {eff }}\left(\frac{4 \pi m \varphi}{M^{2}}\right)\left(\partial_{\mu} \varphi\right)^{2}-\frac{M^{4}}{16 \pi^{2}} \mathcal{V}_{\text {eff }}\left(\frac{4 \pi m \varphi}{M^{2}}\right)$,

where $\mathcal{Z}_{\text {eff }}$ and $\mathcal{V}_{\text {eff }}$ are dimensionless functions (in weak coupling, such actions were considered in Refs. [19-21]). In the weak coupling regime $m \varphi<M^{2} / 4 \pi, \mathcal{V}_{\text {eff }}$ is a power series in $\left[(4 \pi m \varphi) / M^{2}\right]$ and reduces to a quadratic potential with small corrections discussed in Refs. [5,10,22]. The kinetic corrections are also small, reproducing the classic scenario in Ref. [1]. If the last $60 e$ folds of inflation occur in this regime, the tensor-scalar ratio $r \simeq 0.16$ is in conflict with observations [23].

Observations thus force our model to reside at strong coupling $M^{2} / 4 \pi<m \varphi$ during the epoch at which the observed CMB fluctuations are generated. The effective theory remains valid at least to $m \varphi<M^{2}$, leaving a window in which strongly coupled inflation can occur without exciting UV d.o.f. In this regime, the potential can leave the quadratic regime and flatten out $[3,4,13,14]$. The precise form of flattening will be determined by the UV completion, which fixes the forms of $\mathcal{Z}_{\text {eff }}$ and $\mathcal{V}_{\text {eff }}$. Note that the slow-roll parameters

$$
\begin{aligned}
& \epsilon=\frac{m_{\mathrm{Pl}}^{2}}{2}\left(\frac{V_{\mathrm{eff}}^{\prime}}{V_{\mathrm{eff}}}\right)^{2} \sim\left(\frac{4 \pi m m_{\mathrm{Pl}}}{M^{2}}\right)^{2}, \\
& \eta=m_{\mathrm{Pl}}^{2}\left|\frac{V_{\mathrm{eff}}^{\prime \prime}}{V_{\mathrm{eff}}}\right| \sim\left(\frac{4 \pi m m_{\mathrm{Pl}}}{M^{2}}\right)^{2}
\end{aligned}
$$

are small so long as $\left[\left(4 \pi m m_{\mathrm{Pl}}\right) / M^{2}\right] \ll 1$ : slow roll is compatible with strong coupling for super-Planckian excursions of $\varphi$. The standard slow-roll equations of motion give $16 \pi^{2} X / M^{4} \sim \mathcal{O}(\epsilon)$, so that this phase is self-consistent; we can approximate $\mathcal{K}$ by the linear term.

One variant of flattening is induced by wave function renormalization of the inflaton. For example, if the potential for $\phi$ is quadratic, then the canonically normalized $\chi=\int d \varphi \mathcal{Z}_{\text {eff }}^{1 / 2}\left[(4 \pi m \varphi) / M^{2}\right]$ generically has an effective 
potential of the form $V_{\text {eff }} \sim \chi^{p}, p<2$. This behavior is generic for other $\phi$ potentials too-during a stage of slow-roll inflation, generic $V_{\text {eff }}$ and $\mathcal{Z}_{\text {eff }}$ can be approximated by power laws which get flattened by wave function renormalization. When the strong coupling regime can be approximated by power laws, then $N_{\text {total }} \sim \int d \chi\left[V_{\text {eff }} /\left(m_{\mathrm{Pl}}^{2} \partial_{\chi} V_{\text {eff }}\right)\right] \sim\left(\chi / m_{\mathrm{Pl}}\right)^{2}$. If $M^{2} / m>\chi>$ $M^{2} /(4 \pi m)$, this regime supports $N_{\text {strong }} \lesssim\left(M^{2} / m m_{\mathrm{Pl}}\right)^{2} \simeq$ $(4 \pi)^{2} N_{\text {weak }} \quad e$ folds, where $N_{\text {weak }} \lesssim\left[1 /\left(64 \pi^{2}\right)\right] \times$ $\left[M^{4} /\left(m^{2} m_{\mathrm{Pl}}^{2}\right)\right]$ is the number of $e$ folds in weak coupling. So long as $N_{\text {weak }} \gtrsim 1, N_{\text {strong }} \gtrsim(4 \pi)^{2} \sim 150$. Requiring $N_{\text {weak }}<50$ implies $m^{2}>\left[M^{4} /\left(3200 \pi^{2} m_{\mathrm{Pl}}^{2}\right)\right]$. Demanding slow roll (5) in the strong coupling regime gives $\partial_{\chi}^{2} V_{\text {eff }}<V_{\text {eff }} / 3 m_{\mathrm{Pl}}^{2}$, which yields

$$
\frac{M^{4}}{3200 \pi^{2} m_{\mathrm{Pl}}^{2}}<m^{2}<\frac{M^{4}}{48 \pi^{2} m_{\mathrm{Pl}}^{2}} .
$$

Inflation proceeds from strong coupling to weak coupling; the exact duration of the weak coupling regime is controlled by $m$, but is shorter than $50 e$ folds if $m$ obeys Eq. (6). This guarantees that the observable $\mathrm{CMB}$ anisotropies were generated during the strong coupling regime, such that $r \lesssim 0.1$. However, since the spectral index for the potential $V_{\text {eff }} \sim \chi^{p}$ is $n_{s}=1-[(p+2) /(2 \mathcal{N})]$ at $\mathcal{N} e$ folds before the end of inflation, in this regime $p$ cannot be arbitrarily small in order to fit the observations of $n_{s} \simeq 0.96$ for $50 \lesssim \mathcal{N} \lesssim 60$.

A more extreme reduction of $r$ can occur if in the approximation of the potential $\mathcal{V}_{\text {eff }}$ there are cancellations which cause the potential at large $\phi$ to reach a plateau. This may happen in strongly coupled regimes with many particles, as in the concrete example of Ref. [17] relevant for monodromy models. In that example, $\mathcal{V}_{\text {eff }} \sim$ $\left(1-\left\{1 /\left[1+\left(4 \pi c m \varphi / M^{2}\right)^{p}\right]\right\}\right), c=\mathcal{O}(1)$. Fitting to data implies $\left[M^{2} /(4 \pi \mathrm{cm})\right] \lesssim \mathcal{O}(1) m_{\mathrm{Pl}}$ [18]. Demanding $n_{s} \sim$ 0.96 yields a small tensor-to-scalar ratio, $r \sim$ few $\times 10^{-4}$ [17]. The field variation during inflation is also small, $\delta \varphi \lesssim \mathcal{O}(1) m_{\mathrm{Pl}}$. Such models appear perfectly consistent with observations, although their values of $r$ might be too small to be observable by the next generation of polarization experiments. (Other possibilities involve potentials of the form $A+B \varphi^{p}$ with $A, B$ positive. This allows a wider range of values of $r, n_{s}$ consistent with observation, but requires additional d.o.f. to discharge $A$ and reheat, so we leave this possibility aside. The phenomenology of such potentials was recently revisited in Ref. [24].)

A second phase, also yielding a reduction of $r$, occurs when higher-derivative operators become important, yielding a variant of $k$ inflation. The cosmological observables in $k$ inflation have been studied extensively in Refs. $[16,25,26]$. The higher derivatives modify both the linear and nonlinear perturbations [16]. From the terms in the action quadratic in fluctuations, one finds that the speed of sound of the scalar perturbations $c_{s}$ is different from unity, so that the tensor-scalar ratio is $r=16 c_{s} \epsilon$, where $\epsilon$ is the usual slow-roll parameter. (For small $c_{s}$, it may be important to consider corrections logarithmic in $c_{s}$ [27].) The inflationary consistency relation is $r=-8 c_{s} n_{T}$, where $n_{T}$ is the tensor spectral index. In essence, the higher derivatives improve the slow roll in the scalar field equation, without changing the gravitational field equations; this is reflected in the modification of the consistency condition. Meanwhile, terms in the action that are cubic in fluctuations $[25,26]$ can lead to non-negligible equilateral non-Gaussianities. We will present the results of the numerical investigation of monodromy $k$ inflation to illustrate the tension between small $r$, a spectral index consistent with observations, and small non-Gaussianities in this framework. This tradeoff is the main observational implication of monodromy in this regime.

We will take inflation to be potential driven, as required by the combination of slow roll and the null energy condition. We consider the case in which the potential has a wide, smooth regime of variation towards the minimum instead of a sharply ending plateau. Furthermore, we focus on the regime in which higherderivative terms dominate over the quadratic ones in controlling both the background and the perturbations. Finally, for illustrative purposes, we consider the specific kinetic energy function $K=\mathcal{Z} X+\tilde{\mathcal{Z}}\left[\left(16 \pi^{2} X^{2}\right) / M^{4}\right]$.

In strong coupling, the zero modes satisfy $16 \pi^{2} X / M^{4} \gtrsim 1$ and $16 \pi^{2} V_{\text {eff }} / M^{4} \gtrsim 1$. We switch back to $V_{\text {eff }}$ as opposed to $\mathcal{V}_{\text {eff }}$ for convenience. The background field equations are

$$
3 m_{\mathrm{Pl}}^{2} H^{2}=V_{\text {eff }}, \quad 6 H \dot{\varphi} \tilde{\mathcal{Z}} X=-4 \pi M^{2} m V_{\mathrm{eff}}^{\prime} .
$$

There are a number of possible histories depending on how the derivative terms evolve relative to the potential. We will explore this in detail and summarize the results here. If the derivatives decay faster than the potential, higher-derivative inflation turns over to inflation with quadratic kinetic terms after some number of $e$ folds controlled by $m$. If the derivatives decay more slowly than the potential or grow, higher-derivative inflation can continue on all the way to the boundary of strong coupling. At this point $16 \pi^{2} X / M^{4}>1$, but $16 \pi^{2} V_{\text {eff }} / M^{4}=1$, and the slow-roll conditions will be violated. If $m$ is too large, after the transition to weak coupling, the potential will be unable to support any slow roll, and inflation will end. For smaller $m$, after the transition to weak coupling, the dynamics will dissipate the derivatives, and slow-roll inflation with a quadratic potential can restart. Inflation therefore can have at least two stages: a strongly coupled slow roll with large derivatives, and a short weakly coupled stage with small derivatives (whose duration may be so short as to be negligible, depending on $m$ ). The weakly coupled regime must be shorter than $50 e$ folds in order to allow strong coupling effects to flatten the potential and reduce $r$ to meet 
the observational bounds, implying that $m$ still must satisfy Eq. (6). Happily, suppression of $r$ by higher derivatives can occur without increasing $n_{s}$ relative to weakly coupled inflation, which allows a theory consistent with observations. However, nonlinearities induced by higher derivatives yield non-Gaussianities; this in turn again yields a lower bound on $r$ in this regime.

We consider the effective theory for the inflaton as in Ref. [28], neglecting the mixing with gravity. This is a good approximation for energies $E \gg \sqrt{\epsilon} H$ and $c_{s}^{2} \gg \epsilon$, and it yields an almost scale-invariant spectrum of fluctuations with small non-Gaussianities, as observations require. In this limit, working in the gauge where the spatial metric is unperturbed and flat, the field perturbation $\pi$ is defined by $\varphi(t, \vec{x})=\varphi_{0}[t+\pi(t, \vec{x})]$; the spatial curvature perturbation is $\mathcal{R}=-H \pi$. Expanding Eq. (3) up to third order, and keeping only the terms lowest in derivatives, we get

$$
\begin{aligned}
S= & -\int d t d^{3} \vec{x} a^{3} m_{\mathrm{Pl}}^{2} \dot{H}\left[\frac{1}{c_{s}^{2}} \dot{\pi}^{2}-\frac{\left(\partial_{i} \pi\right)^{2}}{a^{2}}+\left(\frac{1}{c_{s}^{2}}-1\right)\right. \\
& \left.\times\left(\dot{\pi}^{3}+\frac{2}{3} c_{3} \dot{\pi}^{3}-\dot{\pi} \frac{\left(\partial_{i} \pi\right)^{2}}{a^{2}}\right)\right] .
\end{aligned}
$$

In terms of the EFT of monodromy (3), the speed of sound is $c_{s}^{2}=\partial_{X} \mathcal{K} /\left(\partial_{X} \mathcal{K}+2 X \partial_{X}^{2} \mathcal{K}\right), \quad$ and $\quad c_{3}\left(1 / c_{s}^{2}-1\right)=$ $2 X^{2} \partial_{X}^{3} \mathcal{K} / \partial_{X} \mathcal{K}$. When $X>\left[M^{4} /\left(16 \pi^{2}\right)\right]$, this means $c_{s}^{2} \sim$ $\left[M^{4} /\left(32 \pi^{2} X\right)\right]\left(\mathcal{K}^{\prime \prime} / \mathcal{K}^{\prime}\right)$ and $c_{3}\left(1 / c_{s}^{2}-1\right)=\left[\left(512 \pi^{4} X^{2}\right) / M^{8}\right]$ $\left(\mathcal{K}^{\prime \prime \prime} / \mathcal{K}^{\prime}\right)$, or $\quad c_{3}=\left[\left(16 \pi^{2} X\right) / M^{4}\right]\left(\mathcal{K}^{\prime \prime \prime} / \mathcal{K}^{\prime \prime}\right)$. Whether $\mathcal{K}$ and its derivatives are $\mathcal{O}(1)$, large, or small when $y=$ $16 \pi^{2} X / M^{4}>1$ depends on the UV theory governing the large- $y$ asymptotics.

The perturbation amplitude leading to CMB fluctuations is set at horizon crossing $\omega \simeq H$, and the scale at which the background breaks time-translation symmetry is $f_{\pi} \propto \sqrt{\dot{\varphi}}$. In the strong coupling regime with large derivative terms, $f_{\pi}^{4}=2 m_{\mathrm{Pl}}^{2} \dot{H} c_{s}$, and [using Eq. (7)] $m_{\mathrm{Pl}}^{2} H^{2} \simeq\left[M^{4} /\left(48 \pi^{2}\right)\right]$. The Gaussian scalar power spectrum is $\Delta_{\mathcal{R}}^{2} \propto\left(H / f_{\pi}\right)^{2}$. $2 \rightarrow 2$ scattering of perturbations shows that the unitarity of Eq. (8) is saturated at $\Lambda^{4}(t) \simeq 16 \pi^{2} m_{\mathrm{Pl}}^{2} \dot{H} c_{s}^{5} /\left(1-c_{s}^{2}\right)$. When $X>M^{4} / 16 \pi^{2}$ in strong coupling, $c_{s}^{2} \lesssim 1$. In this regime, the higher-derivative terms are important both for perturbations and for the background evolution.

Note that our class of monodromy EFTs coupled to gravity can remain valid even when the Goldstone theory (8) breaks down. This could happen when $\epsilon / c_{s}^{2}$ is large, or when the cutoff $\Lambda(t)$ of the action (8) drops below the fundamental cutoff $M$ and even below $f_{\pi}$ for small $c_{s}$. The former requires the inclusion of graviton modes; the latter requires that (8) should be UV-completed by our EFTs for energies $\Lambda<E<M$. Nonetheless, (8) remains valid for $c_{s}$ down to $\sim \epsilon^{1 / 2}$, and for energy scales $\omega<\Lambda(t)$. As long as $H / \Lambda(t) \simeq\left\{\left[3 /\left(16 \pi^{2} \epsilon c_{s}^{5}\right)\right]\left(H / m_{\mathrm{Pl}}\right)^{2}\right\}^{1 / 4}<1$, Eq. (8) can be invoked to describe the horizon crossing of perturbations, allowing (8) to $c_{s} \gtrsim$ few $\times 10^{-2}$. A more detailed treatment is required to fully describe the generation of fluctuations. The concerns about strong coupling for Eq. (8) when $\Lambda<f_{\pi}$ [27] are addressed for us by the full covariant EFT of monodromy, which is valid and under control all the way to the real cutoff $M$, with $f_{\pi}<M$.

The leading non-Gaussianities come from the three-point function. There are two operators in Eq. (8) which source them, leading to three-point functions of the form

$$
\begin{aligned}
\left\langle\Phi_{\vec{k}_{1}} \Phi_{\vec{k}_{2}} \Phi_{\vec{k}_{3}}\right\rangle= & (2 \pi)^{3} \delta_{D}\left(\vec{k}_{1}+\vec{k}_{2}+\vec{k}_{3}\right) \frac{6 \Delta_{\Phi}^{2}}{\left(k_{1}+k_{2}+k_{3}\right)^{3}} \\
& \times\left[f_{\mathrm{NL}}^{(1)} F_{1}\left(k_{1}, k_{2}, k_{3}\right)+f_{\mathrm{NL}}^{(2)} F_{2}\left(k_{1}, k_{2}, k_{3}\right)\right],
\end{aligned}
$$

where

$$
\begin{aligned}
f_{\mathrm{NL}}^{(1)} & =-\frac{85}{324}\left(\frac{1}{c_{s}^{2}}-1\right), \\
f_{\mathrm{NL}}^{(2)} & =-\frac{10}{243}\left(1-c_{s}^{2}\right)\left(\frac{3}{2}+c_{3}\right) .
\end{aligned}
$$

Here $F_{1}$ and $F_{2}$ are induced by $\dot{\pi}(\partial \pi)^{2}$ and $\dot{\pi}^{3}$, respectively [23,26,29].

In our simple example, $c_{3}=0$. More generally, while $c_{3}$ will be of order $1 / c_{s}^{2}$, unless $\mathcal{K}^{\prime \prime \prime} / \mathcal{K}^{\prime \prime} \gg \mathcal{K}^{\prime} / \mathcal{K}^{\prime \prime}$, the numerical prefactors in $f_{\mathrm{NL}}^{(1)}$ are larger than those in $f_{\mathrm{NL}}^{(2)}$ by a factor of 5. A lower bound on $c_{s}$ restricts the range of cubic non-Gaussianities to a narrow strip in the $f_{\mathrm{NL}}^{(1)}, f_{\mathrm{NL}}^{(2)}$ plane [30], similarly to the models of Refs. [31-33].

Current observations may already cut into the space of our strongly coupled EFTs. If we use $c_{s}^{2}=$ $\partial_{X} \mathcal{K} /\left(\partial_{X} \mathcal{K}+2 X \partial_{X}^{2} \mathcal{K}\right)$, we could attain $c_{s}$ as low as $1 / \sqrt{32 \pi^{2}} \simeq 0.056$, when $X \lesssim M^{4}$, while the observational bound $f_{\mathrm{NL}}^{(1)} \lesssim 50$ implies $c_{s} \gtrsim 0.07$ [23]. However, for so small $c_{s}$, the perturbative description of fluctuations using Eq. (8) may be unreliable; yet the full theory may be valid, since $X \lesssim M^{4}$. Thus, a more complete understanding of the perturbations in this regime is needed, and our full theory provides a good starting point for developing it. In Figs. 1 and 2 we present the predictions for the observables $r, n_{s}$, and $f_{\mathrm{NL}}$, for generic power-law potentials in the regimes where Eq. (8) holds. We stress that the precise form of the potential and higher-derivative operators depends on the details of the UV completion. From a bottom-up point of view, we would use data to determine whether our EFT is realized, and given this class of theories, to identify the dominant operators during inflation.

In summary, we have shown that strong coupling dynamics of flux monodromy based on massive 3-form gauge theories naturally leads to flattened potentials at large field values, which are perturbatively and nonperturbatively 


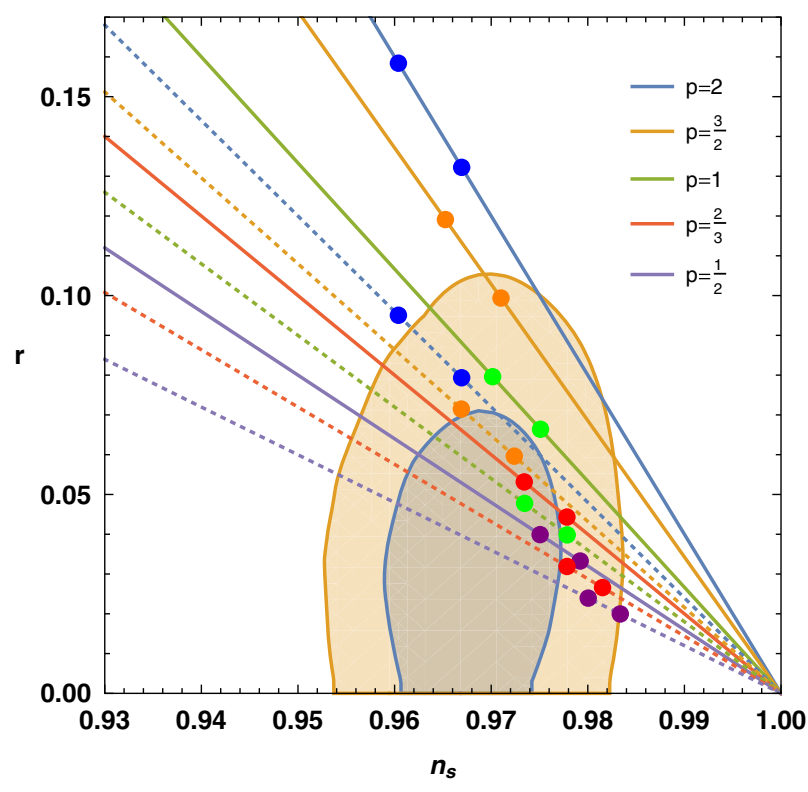

FIG. 1. Planck constraints in the $n_{s}-r$ plane for the $\Lambda \mathrm{CDM}$ model for $V_{\text {eff }} \sim \phi^{p}$, with $p$ 's listed in the legend, and a quartic kinetic term. The solid lines denote $c_{s}=1$; the dashed lines $c_{s}=0.6$. The colored circles denote normalizations at 50 (left) and $60 e$ folds (right).

stable. A key feature of the dynamics is that the curvature of the potential remains small even for large field values. This follows from gauge symmetries of the theory that restrict the potential to the form given in Eq. (3), where the mass

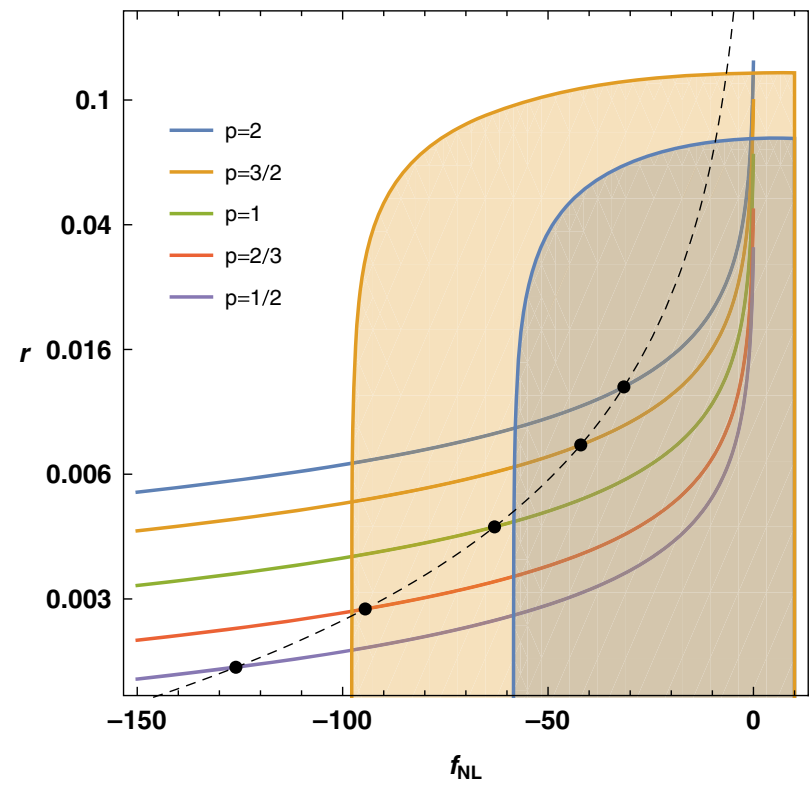

FIG. 2. Tensor-to-scalar ratio $r$ vs equilateral non-Gaussianity $f_{\mathrm{NL}}$ for inflation with $V_{\text {eff }} \sim \phi^{p}$, with fixed $p$ as indicated in the legend; $c_{s}$ varies along each curve from unity at the top right to small values on the left. The kinetic term is quartic in fields. Above the dashed line, $c_{s}^{2} \sim \epsilon$, and the Goldstone action (8) is not a reliable description of perturbations. parameter $m$ is stable since it is also the gauge coupling. For sufficiently low $m$, the slow-roll conditions can be realized naturally, evading the problems of modular inflation [34]. In the case (4), flattening consistent with largish $r$ such as given by power-law potentials either tends to raise the spectral index $n_{s}$ above current bounds, or it gives a plateau potential that meets all observational bounds but predicts a very small $r$.

However, our EFT has a phase which combines both strongly coupled large-field chaotic inflation and $k$ inflation, in which the total reduction of the primordial tensor amplitude arises due to both flattening and higher derivatives. In turn, large derivative contributions increase nonGaussianities, and so in this case satisfy the observational bound $r \lesssim 0.1$ on tensors, while retaining $n_{s}$ near 0.96 requires equilateral non-Gaussianity $f_{\mathrm{NL}}^{e q} \simeq \mathcal{O}(1)$, close to the current observational bounds. This still leaves a broad range of possible values for $r$ that can be probed by future observations, making our natural monodromy models an excellent benchmark.

We would like to thank Zackaria Chacko, Roni Harnik, Matt Kleban, Eva Silverstein, and Alexander Westphal for useful conversations on this and related subjects. N. K. thanks the CERN Theory Division for hospitality in the course of this work. A. L. thanks Nordita and the ICTS for hospitality during the course of this work. N.K. is supported in part by DOE Grant No. DE-SC0009999. A. L. is supported in part by DOE Grant No. DESC0009987.

*damico.guido@gmail.com

†kaloper@physics.ucdavis.edu

*albion@brandeis.edu

[1] A. D. Linde, Chaotic inflation, Phys. Lett. 129B, 177 (1983).

[2] K. Freese, J. A. Frieman, and A. V. Olinto, Natural Inflation with Pseudo-Nambu-Goldstone Bosons, Phys. Rev. Lett. 65, 3233 (1990).

[3] E. Silverstein and A. Westphal, Monodromy in the CMB: Gravity waves and string inflation, Phys. Rev. D 78, 106003 (2008).

[4] L. McAllister, E. Silverstein, and A. Westphal, Gravity waves and linear inflation from axion monodromy, Phys. Rev. D 82, 046003 (2010).

[5] N. Kaloper and L. Sorbo, A Natural Framework for Chaotic Inflation, Phys. Rev. Lett. 102, 121301 (2009).

[6] N. Kaloper, A. Lawrence, and L. Sorbo, An ignoble approach to large field inflation, J. Cosmol. Astropart. Phys. 03 (2011) 023.

[7] N. Kaloper and A. Lawrence, London equation for monodromy inflation, Phys. Rev. D 95, 063526 (2017).

[8] F. Marchesano, G. Shiu, and A. M. Uranga, F-term axion monodromy inflation, J. High Energy Phys. 09 (2014) 184. 
[9] A. Hebecker, F. Rompineve, and A. Westphal, Axion monodromy and the weak gravity conjecture, J. High Energy Phys. 04 (2016) 157.

[10] N. Kaloper and A. Lawrence, Natural chaotic inflation and ultraviolet sensitivity, Phys. Rev. D 90, 023506 (2014).

[11] A. Manohar and H. Georgi, Chiral quarks and the nonrelativistic quark model, Nucl. Phys. B234, 189 (1984).

[12] B. M. Gavela, E. E. Jenkins, A. V. Manohar, and L. Merlo, Analysis of general power counting rules in effective field theory, Eur. Phys. J. C 76, 485 (2016).

[13] X. Dong, B. Horn, E. Silverstein, and A. Westphal, Simple exercises to flatten your potential, Phys. Rev. D 84, 026011 (2011).

[14] L. McAllister, E. Silverstein, A. Westphal, and T. Wrase, The powers of monodromy, J. High Energy Phys. 09 (2014) 123.

[15] C. Armendariz-Picon, T. Damour, and V. F. Mukhanov, $k$-inflation, Phys. Lett. B 458, 209 (1999).

[16] J. Garriga and V. F. Mukhanov, Perturbations in $k$-inflation, Phys. Lett. B 458, 219 (1999).

[17] S. Dubovsky, A. Lawrence, and M. M. Roberts, Axion monodromy in a model of holographic gluodynamics, J. High Energy Phys. 02 (2012) 053.

[18] Y. Nomura, T. Watari, and M. Yamazaki, Pure natural inflation, Phys. Lett. B 776, 227 (2018).

[19] S. Dimopoulos and S. D. Thomas, Discretuum versus continuum dark energy, Phys. Lett. B 573, 13 (2003).

[20] F. Takahashi, Linear inflation from running kinetic term in supergravity, Phys. Lett. B 693, 140 (2010).

[21] K. Nakayama and F. Takahashi, Running kinetic inflation, J. Cosmol. Astropart. Phys. 11 (2010) 009.

[22] N. Kaloper and L. Sorbo, Where in the string landscape is quintessence, Phys. Rev. D 79, 043528 (2009).
[23] P. A. R. Ade et al. (Planck Collaboration), Planck 2013 Results: XXIV. Constraints on primordial non-Gaussianity, Astron. Astrophys. 571, A24 (2014).

[24] P. Creminelli, S. Dubovsky, D. López Nacir, M. Simonović, G. Trevisan, G. Villadoro, and M. Zaldarriaga, Implications of the scalar tilt for the tensor-to-scalar ratio, Phys. Rev. D 92, 123528 (2015).

[25] A. Gruzinov, Consistency relation for single scalar inflation, Phys. Rev. D 71, 027301 (2005).

[26] X. Chen, M.-x. Huang, S. Kachru, and G. Shiu, Observational signatures and non-Gaussianities of general single field inflation, J. Cosmol. Astropart. Phys. 01 (2007) 002.

[27] D. Baumann, D. Green, and R. A. Porto, B-modes and the nature of inflation, J. Cosmol. Astropart. Phys. 01 (2015) 016.

[28] C. Cheung, P. Creminelli, A. L. Fitzpatrick, J. Kaplan, and L. Senatore, The effective field theory of inflation, J. High Energy Phys. 03 (2008) 014.

[29] L. Senatore, K. M. Smith, and M. Zaldarriaga, NonGaussianities in single field inflation and their optimal limits from the WMAP 5-year data, J. Cosmol. Astropart. Phys. 01 (2010) 028.

[30] G. D'Amico and M. Kleban, Non-Gaussianity after BICEP2, Phys. Rev. Lett. 113, 081301 (2014).

[31] M. Alishahiha, E. Silverstein, and D. Tong, DBI in the sky, Phys. Rev. D 70, 123505 (2004).

[32] G. D'Amico, R. Gobbetti, M. Schillo, and M. Kleban, Inflation from flux cascades, Phys. Lett. B 725, 218 (2013).

[33] G. D’Amico, R. Gobbetti, M. Kleban, and M. Schillo, Unwinding inflation, J. Cosmol. Astropart. Phys. 03 (2013) 004.

[34] T. Banks, M. Berkooz, S. H. Shenker, G. W. Moore, and P. J. Steinhardt, Modular cosmology, Phys. Rev. D 52, 3548 (1995). 